\title{
Supercharged LHC tackles Universe's big questions
}

\section{A graphical guide to the science ahead at the Large Hadron Collider.}

\section{Elizabeth Gibney}

03 June 2015

Ramped up in power after a two-year upgrade, the world's most powerful particle accelerator is once again doing science. Following its official restart on 3 June, the Large Hadron Collider (LHC) at CERN, Europe's particle-physics lab near Geneva, Switzerland, can smash protons together faster and with higher energies than during its first run, which ended in February 2013. Our graphical guide illuminates the discoveries that could lie ahead in the next run of the LHC.

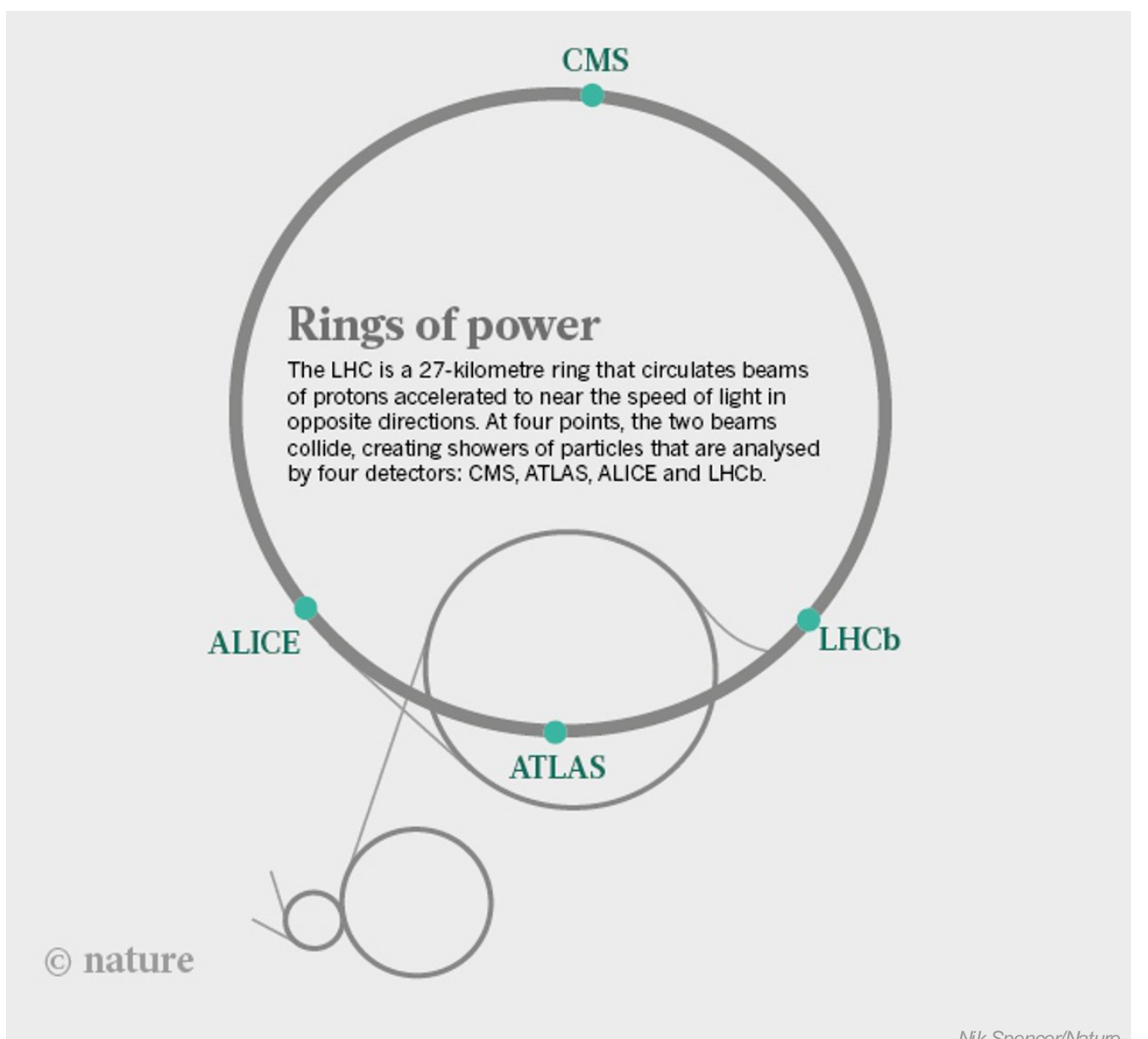

Nik Spencer/Nature

The first run began in earnest in November 2009. The LHC collided particles - mainly protons, but also heavier particles such as lead ions - at high enough energies to confirm the existence of the Higgs boson in 2012, which garnered a Nobel prize for the physicists who had predicted the subatomic particle. 


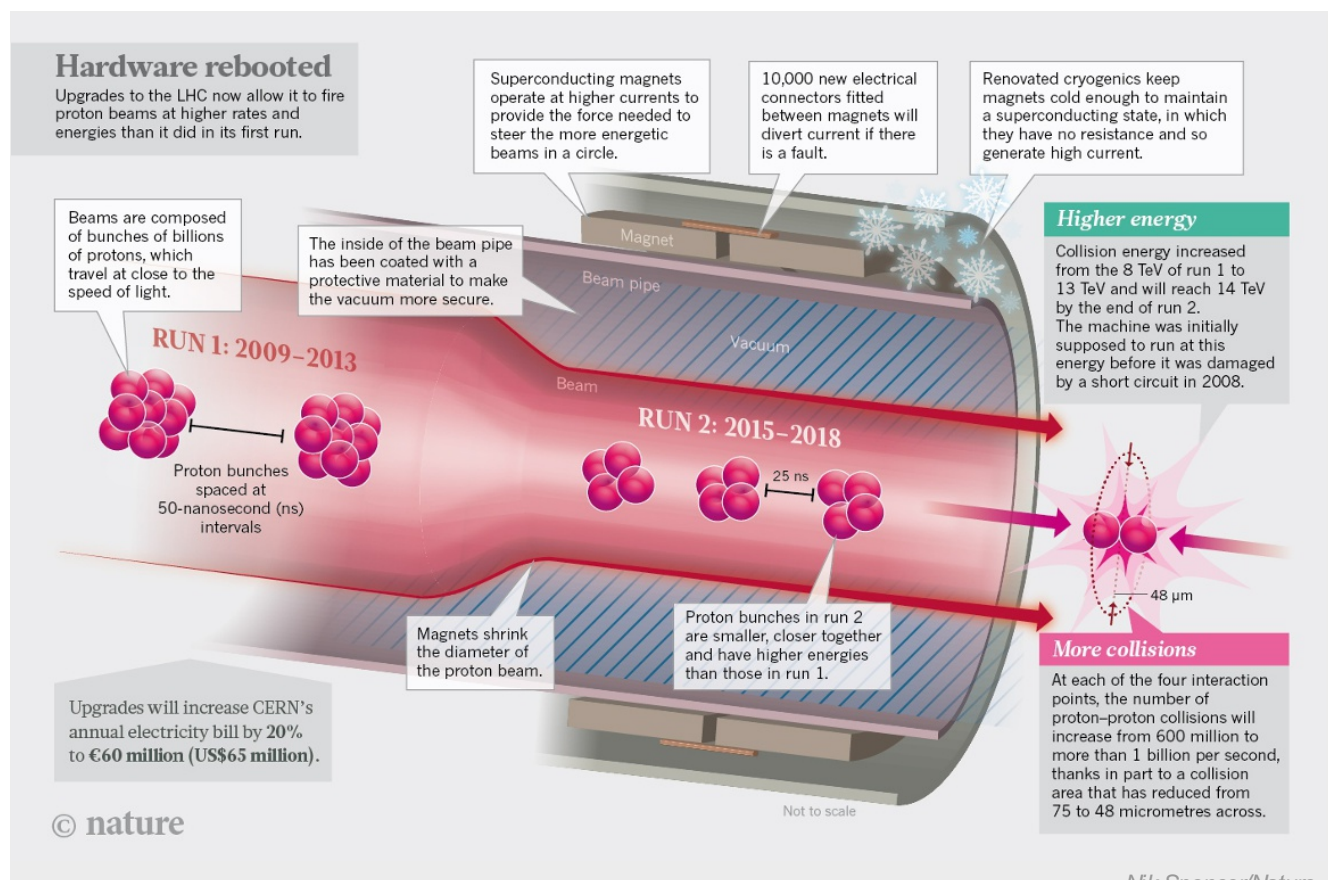

Nik Spencer/Nature

In the next run, set to last three years, energies will rise to an eventual 14 teraelectronvolts (TeV; see 'Hardware rebooted'). One hope is that these high energies will help the LHC to produce evidence for supersymmetry, an elegant theory that could extend the standard model of particle physics. They could also shake out particles of dark matter, the invisible substance that is thought to make up $85 \%$ of the matter in the Universe (see 'Higher energy').

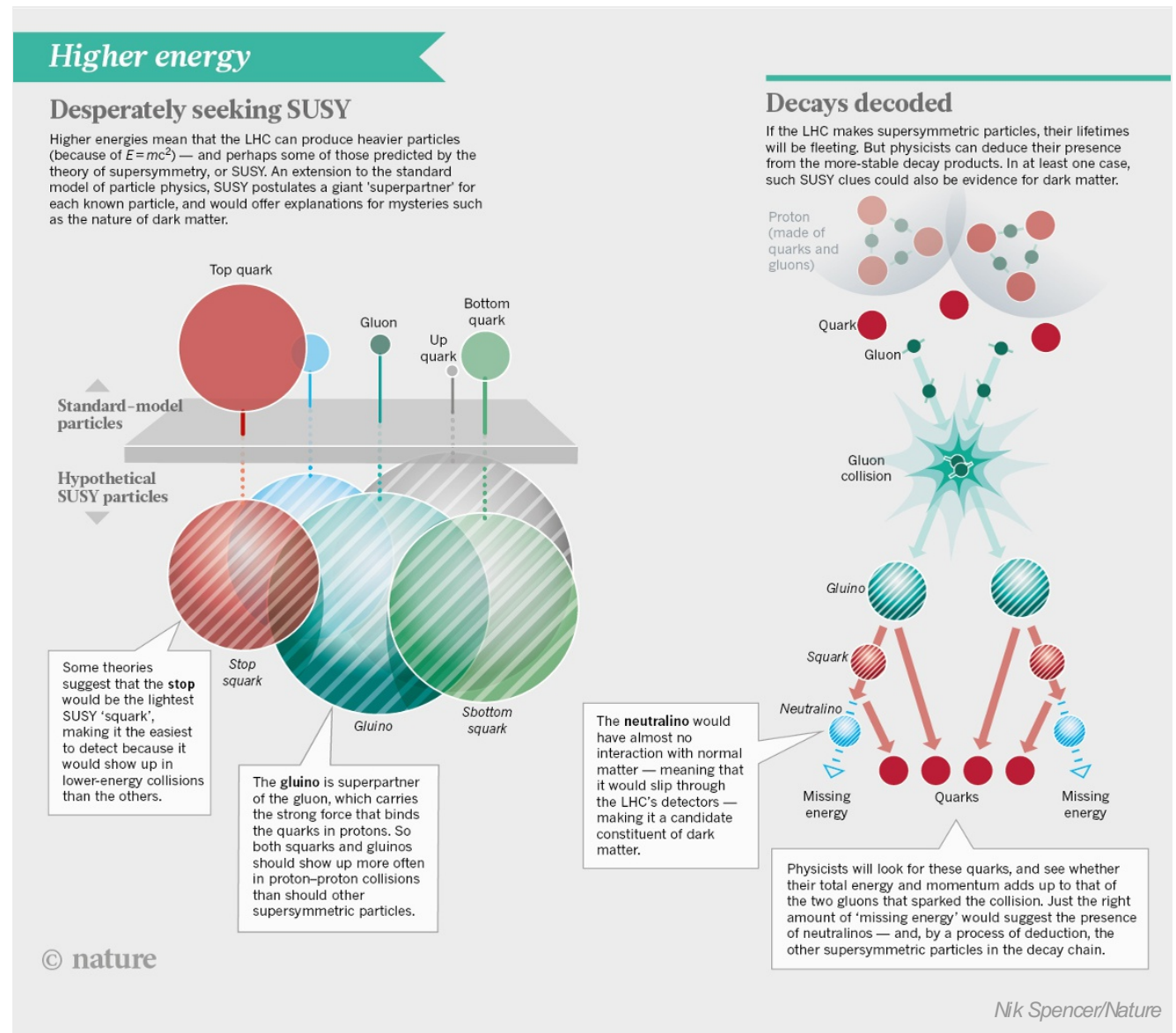

The extra collisions will enable more-precise study of the nature of the Higgs, and will provide clarity on anomalies hinted at in the first run (see 'More collisions'). 


\section{More collisions}

\section{The Higgs factory}

LHC experiments discovered the Higgs boson but they did not produce enough of the particles to examine their properties in much depth.

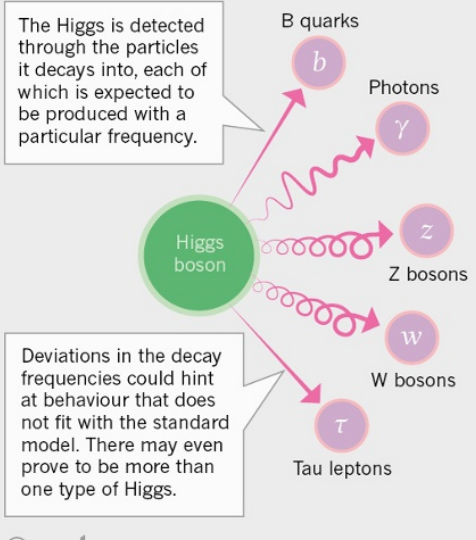

(c) nature

\section{Known unknowns}

More collisions will help to resolve some ongoing mysteries. One of these concerns an anomaly in the way a transient particle called a $\mathrm{B}^{+}$meson decays.

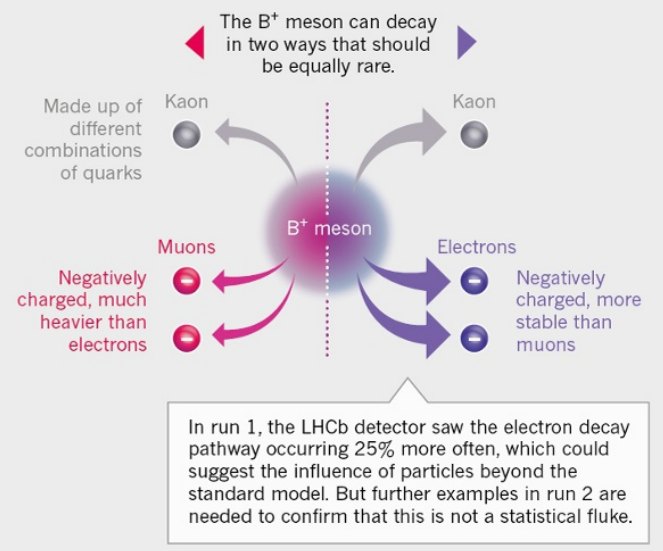

Nik Spencer/Nature

An earlier version of this graphic was published in Nature on 12 March 2015.

Nature | doi:10.1038/nature.2015.17693 